\title{
Remodeling of Synaptic Membranes after Induction of Long-Term Potentiation
}

\author{
Nicolas Toni, Pierre-Alain Buchs, Irina Nikonenko, Patrisia Povilaitite, Lorena Parisi, and Dominique Muller \\ Neuropharmacology, University Medical Center, 1211 Geneva 4, Switzerland
}

Several morphological changes of synapses have been reported to be associated with the induction of long-term potentiation (LTP) in the CA1 hippocampus, including an transient increase in the proportion of synapses with perforated postsynaptic densities (PSDs) and a later occurrence of multiple spine boutons (MSBs) in which the two spines arise from the same dendrite. To investigate the functional significance of these modifications, we analyzed single sections and reconstructed 134 synapses labeled via activity using a calcium precipitation approach. Analyses of labeled spine profiles showed changes of the spine head area, PSD length, and proportion of spine profiles containing a coated vesicle that reflected variations in the relative proportion of different types of synapses. Threedimensional reconstruction indicated that the increase of perforated spine profiles observed $30 \mathrm{~min}$ after LTP induction essentially resulted from synapses exhibiting segmented, com-

An interesting finding of recent years is that synapses are extremely dynamic structures that may change not only their functioning with activity but also their morphology. Analyses of spine dynamics using confocal microscopy showed that these small dendritic protrusions are characterized by continuous shape changes (Fischer et al., 1998) that may depend on neuronal activity (Fischer et al., 2000; Lüscher et al., 2000; Matus, 2000). There is also evidence that increases in intracellular calcium may affect spine morphology (Korkotian and Segal, 1999; Segal et al., 2000). Another interesting possibility is that synaptic activity results in modifications of spine number. Agonist or antagonist modulation of excitatory transmission or even just preparation of hippocampal slices was reported to affect spine density (Kirov and Harris, 1999; Kirov et al., 1999; McKinney et al., 1999). Furthermore, application of high-frequency trains that induce long-term potentiation (LTP) trigger the growth of filopodia (Maletic-Savatic et al., 1999) or even dendritic spines-like structures (Engert and Bonhoeffer, 1999). In agreement with these reports, we found, using an electron microscopic analysis of synapses likely to be activated, that LTP induction resulted in a sequence of morphological changes with a transient increase in synapses with perforated postsynaptic densities (PSDs), followed

Received Jan. 31, 2001; revised May 21, 2001; accepted May 31, 2001.

This work was performed with the support of Grant 31-56852.99 from the Swiss National Science Foundation. We thank M. Moosmayer for excellent technical support, Fred Pillonel for photographic work, and D. Smithies, K. M. Harris, and J. C. Fiala for providing image analysis software.

Correspondence should be addressed to Dr. D. Muller, Neuropharmacologie, Centre Médical Universitaire, 1211 Geneva 4, Switzerland. E-mail: Dominique.Muller@medecine.unige.ch

Copyright (C) 2001 Society for Neuroscience $0270-6474 / 01 / 216245-07 \$ 15.00 / 0$ pletely partitioned PSDs. These synapses had spine head and PSD areas approximately three times larger than those of simple synapses. They contained coated vesicles in a much higher proportion than that of any other type of synapse and exhibited large spinules associated with the PSD. Also the MSBs with two spines arising from the same dendrite that were observed 1-2 $\mathrm{hr}$ after LTP induction included a spine that was smaller and a PSD that was smaller than those of simple synapses. These results support the idea that LTP induction is associated with an enhanced recycling of synaptic membrane and that this process could underlie the formation of synapses with segmented PSDs and eventually result in the formation of a new, immature spine.

Key words: morphology; postsynaptic density; coated vesicles; plasticity; hippocampus; rat by multiple spine boutons (MSBs) in which the two spines arise from the same dendrite (Toni et al., 1999).

Whether and how these morphological changes relate to the increase in synaptic efficacy remain, however, unknown. Among possibilities, one suggestion has been that the synapses with a perforated PSD that were observed initially could represent a morphological correlate of the mechanisms of receptor recycling proposed to contribute to LTP (Lüscher et al., 2000). Evidence from several studies indicates that addition of postsynaptic AMPA receptors to the postsynaptic density is likely to play an important role in changes of synaptic efficacy (Lüscher et al., 2000). Activity modifies the insertion of new receptors at the membrane (Shi et al., 1999; Zhu et al. 2000), and interference via peptides or toxins with the mechanisms of exocytosis or endocytosis affects LTP or LTD induction and AMPA receptormediated synaptic responses (Lledo et al., 1998; Nishimune et al., 1998; Lüscher et al., 1999). Thus postsynaptic exocytotic or endocytotic mechanisms (Maletic-Savatic and Malinow, 1998) could contribute to receptor turnover and changes in synaptic function. Furthermore they could mediate the remodeling of synaptic membranes that underlies the formation of synapses with a perforated PSD. An additional possibility concerning synapses with a perforated PSD is that they represent an intermediary step in a process of spine duplication (Nieto-Sampedro et al., 1982; Carlin and Siekevitz, 1983; but see Sorra et al., 1998).

To understand better the functional implication of the different types of synapses observed after LTP induction and to investigate the possibility that an increased recycling of synaptic membrane takes place, we used electron microscopy and analyzed the properties of 134 reconstructed, labeled synapses. The results are consistent with the idea that LTP induction promotes a remod- 
eling of synaptic membranes that may account for the reported changes in the different types of synapses.

\section{MATERIALS AND METHODS}

Preparation and stimulation of cultures. Hippocampal organotypic slice cultures were prepared from 7-d-old rats and maintained $12 \mathrm{~d}$ in culture as described by Stoppini et al. (1991) before testing in an interface recording chamber. A stimulation electrode made of twisted nichrome wires was placed in the CA3 area, and evoked field potentials were recorded in the CA1. LTP was induced using $\theta$ burst stimulation consisting of five bursts at $5 \mathrm{~Hz}$ with each burst composed of four pulses at $100 \mathrm{~Hz}$. This pattern was applied twice at $10 \mathrm{sec}$ intervals and once again, 5 min before fixation, to relabel the same synapses. All slice cultures processed for EM came from different animals.

Electron microscopy processing. At different times after LTP induction, cultures were fixed and processed for electron microscopy as described previously (Buchs et al., 1994). Briefly, cultures were fixed overnight at

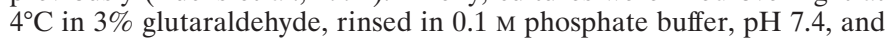
post-fixed $2 \mathrm{hr}$ in a fresh solution of $1 \%$ osmium tetroxide $\left(\mathrm{OsO}_{4}\right)$ with $1.5 \%$ potassium chromium trisoxalate $\left[\left(\mathrm{K}_{3} \mathrm{Cr}\left(\mathrm{C}_{2} \mathrm{O}_{4}\right)\right)_{3}\right.$; Aldrich, Milwaukee, WI], $\mathrm{pH}$ 9.5. After a 5 min rinse in $\mathrm{KOH}, \mathrm{pH} 9.5$, the samples were classically dehydrated in ethanol and embedded in Epon (Fluka, Buchs, Switzerland). For serial EM, ribbons of up to 60 sections were cut in the middle portion of the apical arborization of CA1 pyramidal neurons (ultratome Ultracut-E; Reichert-Jung) and collected on single-slotted Formvar-coated grids (TAAB Laboratories, Aldermaston, UK). Sections were stained for $45 \mathrm{~min}$ in $0.5 \%$ uranyl acetate and $45 \mathrm{sec}$ in lead citrate and analyzed on a Philips CM10 electron microscope at a magnification of $11,000-29,100 \times$.

Morphological analyses. Synapses were defined by the presence of a clear postsynaptic density facing at least three presynaptic vesicles. Perforated synapses were defined by the presence of a discontinuity in the postsynaptic density (Geinisman et al., 1987), and MSBs were defined by the presence of two independent dendritic spines contacting the same axon terminal (Sorra and Harris, 1993).

For two-dimensional morphometrical studies, five to six sections per culture were examined, and a total of 200-1519 synapses out of 4-12 hippocampal slice cultures per time point were analyzed. For each section, all labeled synapses observed in an area corresponding to the middle third of the CA1 stratum radiatum and clearly identified by the presence of a PSD were considered for statistical measurements. Negatives were digitized, and PSD length and cross-sectional area were measured using a software developed by D. Smithies on AVS.

For three-dimensional experiments, a total of 134 synapses were reconstructed out of eight hippocampal slice cultures. Synapses were selected on the test section on the basis of the presence of a precipitate in the postsynaptic spine and the possibility to identify easily the PSD as simple or perforated on one of the serial sections. The profiles were then photographed serially at a magnification of at least $16,000 \times$ and analyzed using a software developed by J. C. Fiala and K. M. Harris. The small-fold procedure was used for determination of section thickness, and an average value of $42 \mathrm{~nm}$ was used for reconstruction. This value and the measurements on serial sections were used for calculating the surface area of PSDs and spine heads.

Data are presented as a mean \pm SEM, with $n$ indicating the number of synapses analyzed. Statistical analyses were performed using the Student's $t$ test or the $\chi^{2}$ test, when stated.

\section{RESULTS}

Organotypic slice cultures prepared from 7-d-old neonate rats were stimulated using $\theta$ burst-patterned stimulation and fixed at different times after LTP induction using a protocol that reveals, in the form of a fine precipitate, the presence of calcium accumulated in subcellular structures. Previous work showed that this approach makes it possible to identify a subset of labeled spine profiles $(\sim 12-15 \%$ of all profiles) likely to represent stimulated synapses (Buchs and Muller, 1996; Toni et al., 1999). In the present study, we analyzed labeled synapses observed 15-30 min and 1-2 hr after LTP induction and compared them with labeled synapses observed 5 min after LTP induction, a time at which morphological modifications are not yet detected, or with labeled synapses obtained from slices stimulated in the presence of $\mathrm{KN}$ -
93, a calcium/calmodulin-dependent protein kinase II antagonist that prevents LTP induction (Toni et al., 1999).

As illustrated in Figure 1, the morphological changes detected after LTP induction consisted of changes in the relative proportion of three distinct synaptic types referred to as simple synapses (synapses with a unique and continuous PSD; Fig. $1 \mathrm{~A}$, left), synapses with perforated PSDs (synapses exhibiting a discontinuity of the PSD; Fig. $1 \mathrm{~A}$, middle), and MSBs (defined by the presence of two spines contacting the same presynaptic terminal; Fig. 1A, right) (Sorra and Harris, 1993). Under control conditions, simple synapses represented the majority of labeled spine profiles (70.4 $\pm 2.3 \% ; n=14 ; 1519$ profiles analyzed; Fig. $1 B)$. Their proportion, however, decreased markedly $30 \mathrm{~min}$ after LTP induction $(46.9 \pm 2.8 \% ; n=4 ; 358$ profiles analyzed; $p<0.01)$, because of an increase in the proportion of synapses with perforated PSDs (from $22.4 \pm 2.3$ to $45.8 \pm 2.8 \% ; n=4 ; 358$ profiles analyzed; $p<0.01$; Fig. $1 B$ ). The proportion of synapses with perforated PSDs completely recovered 1-2 hr after LTP induction, and these were replaced, to some extent, by images of MSBs (from $7.1 \pm 1.2$ to $14.6 \pm 1.5 \% ; n=10 ; 755$ profiles analyzed; $p<$ 0.01; Fig. $1 B$ ). Previous work performed using three-dimensional reconstruction provided evidence that the increase in the proportion of MSBs observed 1-2 hr after LTP induction mainly represented cases in which the two spines arose from the same dendrite, cases that will be referred to as duplicated spines (Toni et al., 1999).

Single-section analyses of parameters of these labeled synapses, such as the spine profile area, PSD length, and presence of coated vesicles, revealed changes as a function of time after LTP induction. As shown on Figure $1 C$, all of these parameters increased 30 min after LTP induction and recovered to control values or close to control values after 1-2 hr. These changes, however, were not caused by modifications in the characteristics of synapses but essentially reflected modifications in the relative proportion of the three types of synapses. Comparison of the spine profile area or PSD length of simple synapses revealed no statistically significant differences over time $\left(0.26 \pm 0.01\right.$ vs $0.29 \pm 0.01 \mu \mathrm{m}^{2}$ and $0.38 \pm 0.01$ vs $0.37 \pm 0.01 \mu \mathrm{m}$, respectively; $n=2-3 ; 131$ and 163 profiles analyzed for spine area and PSD length, under control conditions and at $30 \mathrm{~min}$, respectively). Similarly, the characteristics of spine profiles of synapses with perforated PSDs were comparable under control conditions and at $30 \mathrm{~min}(0.42 \pm 0.02$ vs $0.43 \pm 0.02 \mu \mathrm{m}^{2}$ and $0.51 \pm 0.02$ vs $0.50 \pm 0.01 \mu \mathrm{m} ; n=101$ and 163). Thus two-dimensional analyses did not reveal particular changes in the characteristics of these types of synapses but rather revealed modifications in the proportion of the different types of synapses.

We therefore continued by analyzing their properties using three-dimensional reconstruction on a group of 134 labeled synapses. In Figure 2, we compared the surface area of the spine head, the surface area of the PSD, and the proportion of spines exhibiting coated vesicles in a set of 31 simple synapses and 42 synapses with perforated PSDs. As expected from previous work, synapses with perforated PSDs were characterized by a much larger spine head, a larger PSD area, but also a much higher proportion of spines containing one or several coated vesicles $\left(1.58 \pm 0.19\right.$ vs $0.62 \pm 0.06 \mu \mathrm{m}^{2} ; 0.17 \pm 0.02$ vs $0.07 \pm 0.02 \mu \mathrm{m}$; 44 vs $13 \% ; n=6-8$ slice cultures, 31 and 42 reconstructed synapses). The ratio of these parameters between synapses with perforated PSDs and simple synapses was 2.9, 3.1, and 3.4, respectively. Interestingly, the ratio of the PSD area versus the spine membrane area remains constant between simple synapses 

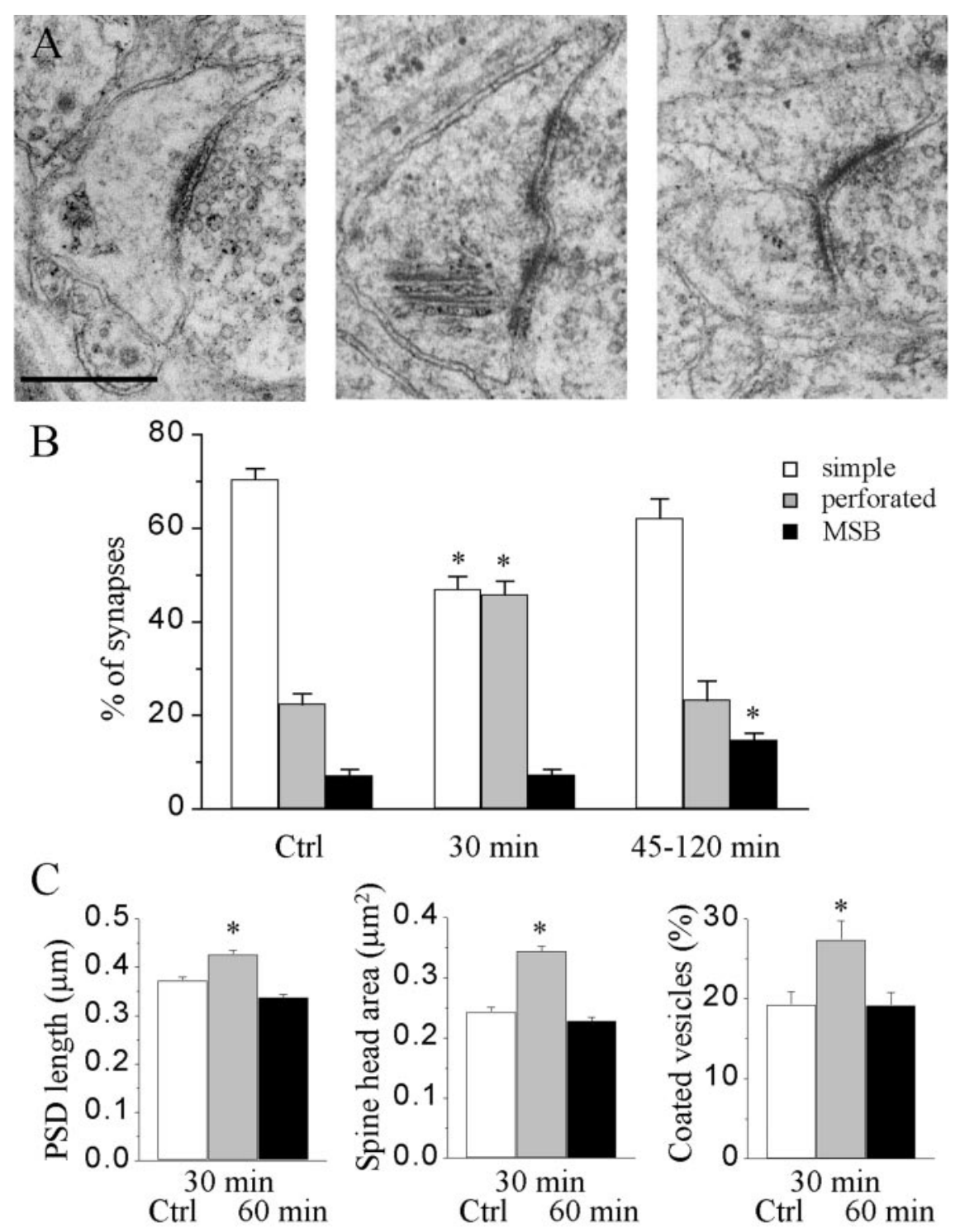

Figure 1. Changes in the proportion of different types of synapses and their characteristics during LTP. $A$, Illustration of a simple synapse with a single PSD (left), a synapse with a perforated PSD (middle), and an MSB (right). Scale bar, $0.5 \mu \mathrm{m}$. B, Proportion of the three types of synapses under control conditions and at 30 and 45-120 min after LTP induction ( $n=4-14$ hippocampal slice cultures and 358-1519 synaptic profiles analyzed; * $p<0.01$ ). $C$, Changes in PSD length (left), spine head profile area (middle), and the proportion of spine profiles containing coated vesicles (right) determined via single-section analysis of the entire population of labeled synapses $(n=4-9$ hippocampal slice cultures and 360-660 synaptic profiles; ${ }^{*} p<0.01$ ). Ctrl, Control. and synapses with perforated PSDs (10.7 vs $11.3 \%$, respectively), despite a marked difference in the size of the spines.

We then investigated whether the characteristics of the synapses with perforated PSDs seen 30 min after LTP induction were similar to those of synapses with perforated PSDs observed under control conditions. For this, we compared the parameters of 20 and 23 synapses with perforated PSDs reconstructed from serial sections of slices fixed either 30 or 5 and 60 min after LTP induction. The 5 and $60 \mathrm{~min}$ time points were used as the control, because the slice cultures have received the same stimulation protocol, but the changes in the proportion of perforated synapses are not detected at those moments. Consistent with the results obtained with two-dimensional analyses, the dimensions of synapses with perforated PSDs (spine area and PSD area) did not vary with time after LTP induction (data not shown). However, as illustrated in Figure 3, the synapses reconstructed 30 min after LTP induction differed from the others in several ways. First, there were differences in the shape of the PSD. Synapses with perforated PSDs have been classified in three major types depending on the organization of the PSD (Fig. 3): fenestrated (with a central, macular zone lacking PSD), horseshoe-shaped (when the perforation reaches the edges of the PSD), or seg- mented (when the PSD is completely partitioned into two distinct zones). As shown on Figure 3B, synapses with perforated PSDs under control condition were mostly of the fenestrated or horseshoe type $(83 \%)$. In contrast however, synapses with perforated PSDs 30 min after LTP induction were mainly of the segmented type (Fig. $3 B ; 45$ vs $17 \% ; p<0.05 ; \chi^{2}$ ). When considering the relative proportion of these different types of synapses with perforated PSDs and the increase observed after LTP induction, it appears that the changes are essentially caused by synapses with segmented, completely partitioned PSDs (Fig. 3C).

A second interesting characteristic of the synapses with perforated, segmented PSDs seen at $30 \mathrm{~min}$ is that they contained in a great proportion one or several coated vesicles (Fig. 4D). In $69 \%$ of synapses with segmented PSDs, coated vesicles could be observed, whereas only $27 \%$ of synapses with perforated, nonsegmented PSDs exhibited such vesicles ( $n=13$ and 30; $p<0.05$ ). Note also that the proportion of reconstructed, simple synapses that contained coated vesicles was even lower $(13 \% ; n=31 ; p<$ $0.01)$. To examine the location within spines of coated vesicles, we then analyzed 452 spine profiles taken at different times after LTP induction. As illustrated in Figure 4, these vesicles were usually located in the center of the spine head ( $50 \%$ of cases), but 

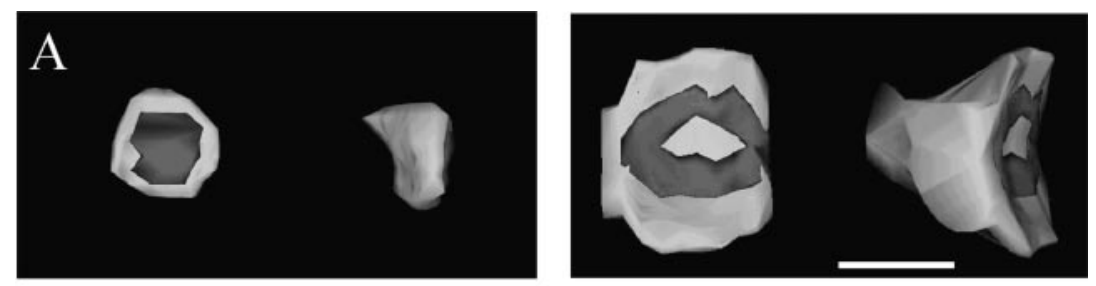

B

Figure 2. Characteristics of three-dimensionally reconstructed simple synapses and synapses with perforated PSDs observed $30 \mathrm{~min}$ after LTP induction. A, Illustration of the spine head of and PSD of a reconstructed simple synapse (left) and a synapse with a perforated PSD (right). Scale bar, $0.5 \mu \mathrm{m}$. B, Values of spine head surface area (left), PSD surface area (middle), and the proportion of spines containing coated vesicles for simple synapses $(\mathrm{Ctrl})$ and synapses with perforated PSDs (Perforated). Data are the mean \pm SEM of measurements made on 31 reconstructed simple synapses and 42 synapses with perforated PSDs $\left({ }^{*} p<0.01\right)$.

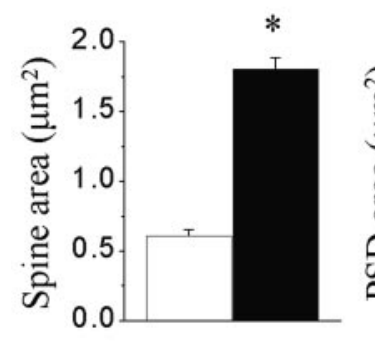

Ctrl Perforated

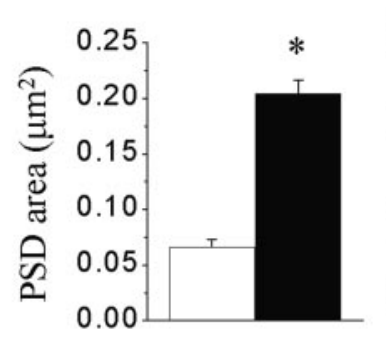

Ctrl Perforated

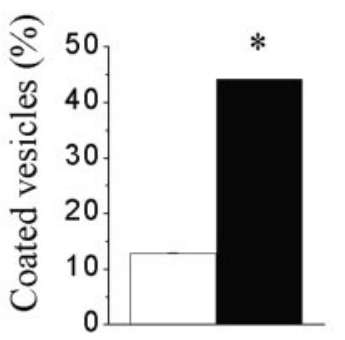

Ctrl Perforated

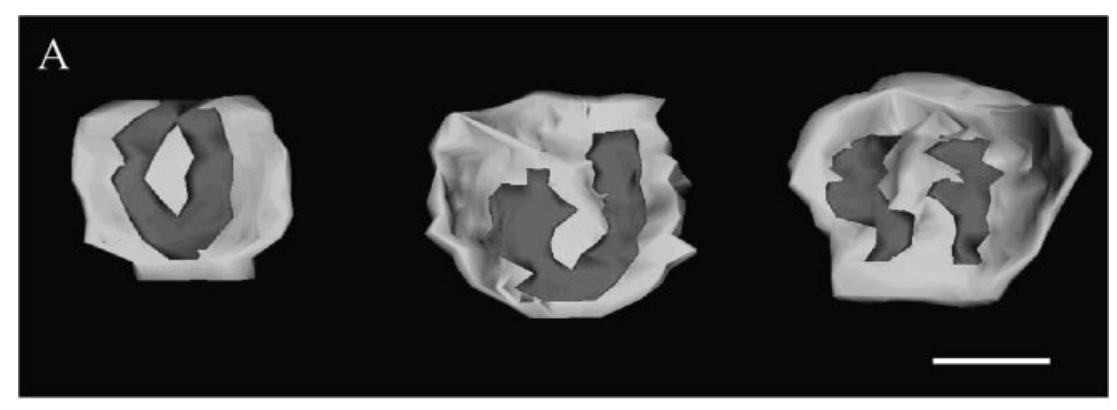

Figure 3. Increase in the proportion of synapses with segmented PSDs 30 min after LTP induction. $A$, Illustration of three types of reconstructed synapses with perforated PSDs. From left to right, synapses with fenestrated, horseshoe-shaped, and segmented PSDs are shown. Scale bar, $0.5 \mu \mathrm{m}$. B, Proportion of the three types of synapses with perforated PSDs under control conditions and $30 \mathrm{~min}$ after LTP $(n=20$ and 23, respectively). The two distributions are statistically significantly different $\left(p<0.05, \chi^{2}\right)$. $C$, Changes in the proportion of synapses with perforated PSDs calculated for synapses with segmented (white column) and nonsegmented (black column) PSDs according to the threedimensional reconstruction. Data are the mean \pm SEM.

B

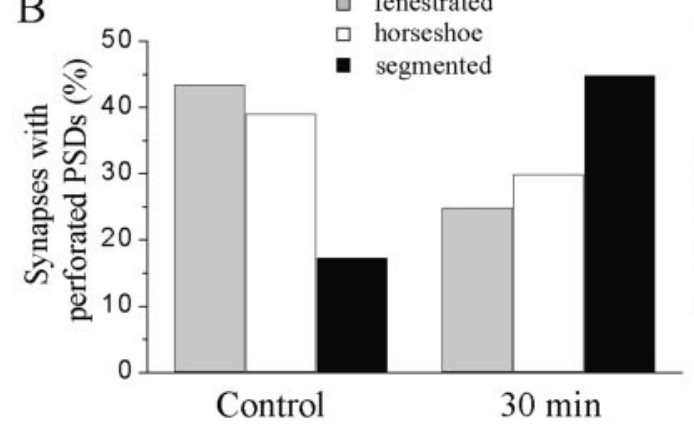

C

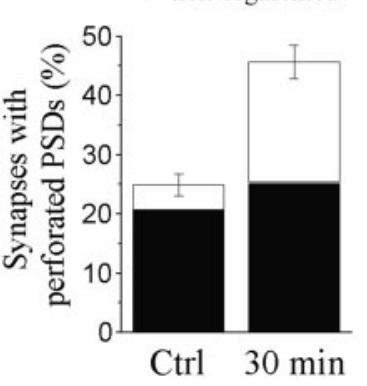

they could also be seen in contact with or emerging from the spine apparatus (6\% of cases), closed to or fusing with the synaptic membrane (44\% of cases), including the area of the PSD (12\% of cases). No significant changes in the distribution of coated vesicles could be detected as a function of time after LTP induction. Thus an important characteristic of the synapses with segmented PSDs observed $30 \mathrm{~min}$ after LTP induction was the presence of an increased proportion of coated vesicles.

A third characteristic concerned the presence and size of spinules in association with the PSD. Spinules are small fingerlike protrusions of the postsynaptic membrane into the presynaptic ending (Fig. $5 A, B$ ). The size of these protrusions was determined by measuring their maximal length, and they were classified as small when $<0.2 \mu \mathrm{m}$ or large for sizes $>0.2 \mu \mathrm{m}$. As shown in Figure $5 C$, the majority of synapses with fenestrated PSDs did not contain spinules, and if present, they were almost systematically of a small size. In contrast, most of the synapses with segmented PSDs, in addition to having clearly separated PSDs, usually also exhibited a spinule in between, which in many cases was characterized by a large size. Synapses with horseshoetype PSDs somehow represented an intermediary stage, because most of them contained a spinule of small size. The three distributions of the three types of synapses with perforated PSDs are statistically significantly different $\left(p<0.05 ; \chi^{2}\right)$. The synapses with perforated PSDs observed $30 \mathrm{~min}$ after LTP were thus particular in that they mostly exhibited segmented PSDs and a higher proportion of them contained coated vesicles and large spinules associated with the PSD.

We then also analyzed the characteristics of MSBs, the third type of synapse found to appear 1-2 hr after LTP induction. Previous work performed using three-dimensional reconstruction showed that the spines contacting such MSBs 1-2 hr after LTP induction originated from the same dendrite and exhibited features of mature synapses (Toni et al., 1999). Comparison of 30 

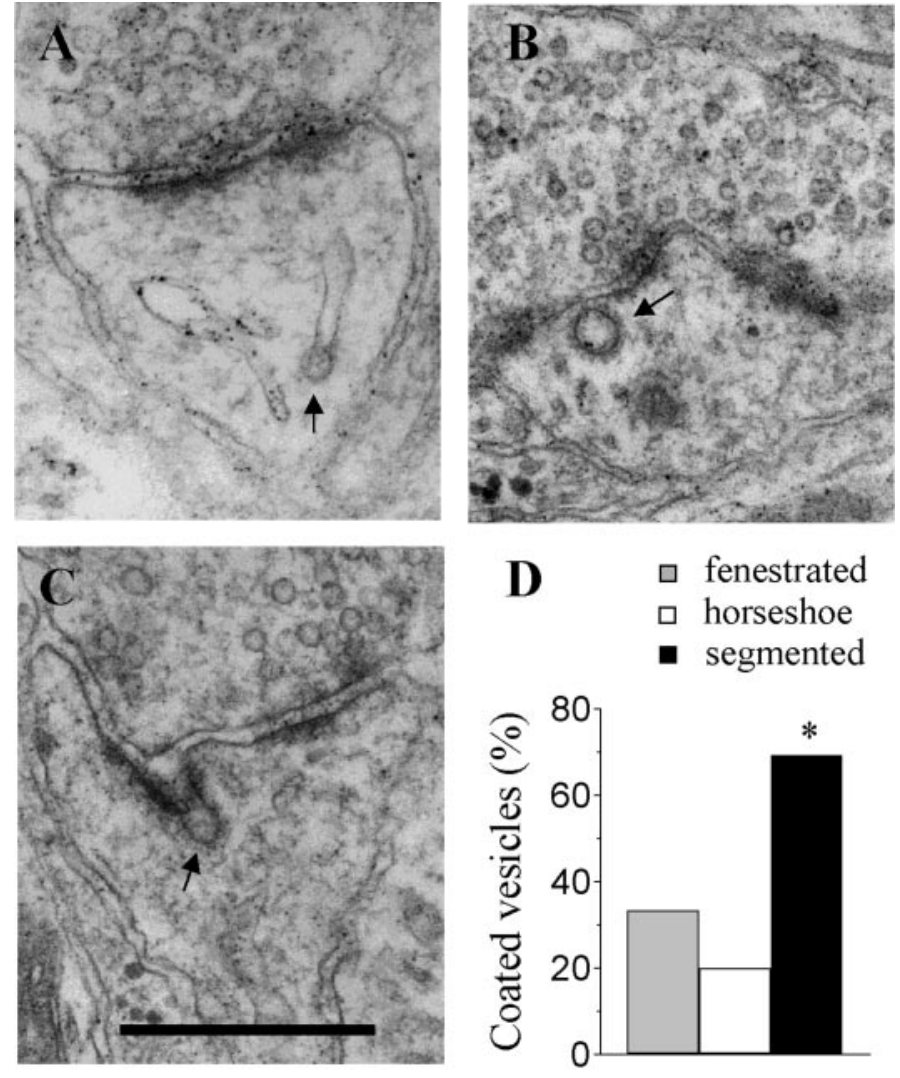

Figure 4. Increased proportion of coated vesicles in synapses with segmented PSDs observed $30 \mathrm{~min}$ after LTP induction. $A$, Example of a coated vesicle emerging from or fusing with the spine apparatus. $B$, Coated vesicle within the spine head. $C$, Example of a coated vesicle seen fusing with the synaptic membrane at the level of the PSD. Scale bar: $A-C, 0.5 \mu \mathrm{m}$. D, Proportion of synapses with fenestrated (gray column), horseshoe-type (white column), and segmented (black column) PSDs that contained one or several coated vesicles in a population of 15,15 , and 13 reconstructed synapses, respectively ( ${ }^{*} p<0.05$ ); arrows in $A-C$ point to examples of coated vesicles.

reconstructed duplicated synapses with 30 reconstructed simple synapses revealed, however, some differences. First, duplicated synapses are characterized by smaller PSDs than are simple synapses. In average, the PSD of a duplicated synapse represented $\sim 76 \%$ of the size of the PSD of a simple synapse (Fig. 6). Interestingly, this difference was related to the fact that duplicated synapses were constituted of two spines with distinct characteristics. The largest of the two spines exhibited a spine head area and PSD area indistinguishable from those of simple synapses (Fig. $6 B, 0.65 \pm 0.05$ vs $0.61 \pm 0.04 \mu \mathrm{m}^{2} ; n=15$ and 31 , for spine head area; $0.070 \pm 0.009$ vs $0.066 \pm 0.06 \mu \mathrm{m}^{2}$ for PSD area). In contrast, however, the smallest of the two spines exhibited a spine head area and PSD area significantly smaller than those of simple synapses $\left(0.48 \pm 0.05\right.$ vs $0.61 \pm 0.04 \mu \mathrm{m}^{2} ; n=15$ and $31 ; p<0.05$ for spine head area; $0.039 \pm 0.006$ vs $0.066 \pm$ $0.006 \mu \mathrm{m}^{2} ; p<0.01$ for PSD area). Interestingly, duplicated synapses very rarely exhibited perforated PSDs. The proportion of perforated PSDs among duplicated synapses was $10 \%$ of the total number of spines $(n=30)$. This value is lower than that of control synapses $(22.4 \pm 2.2 \% ; n=2158)$ or even lower than that of MSBs formed by spines arising from different dendrites $(23 \%$; $n=30)$. These characteristics of duplicated synapses suggest therefore that one of the two spines is still characterized by features of immaturity.
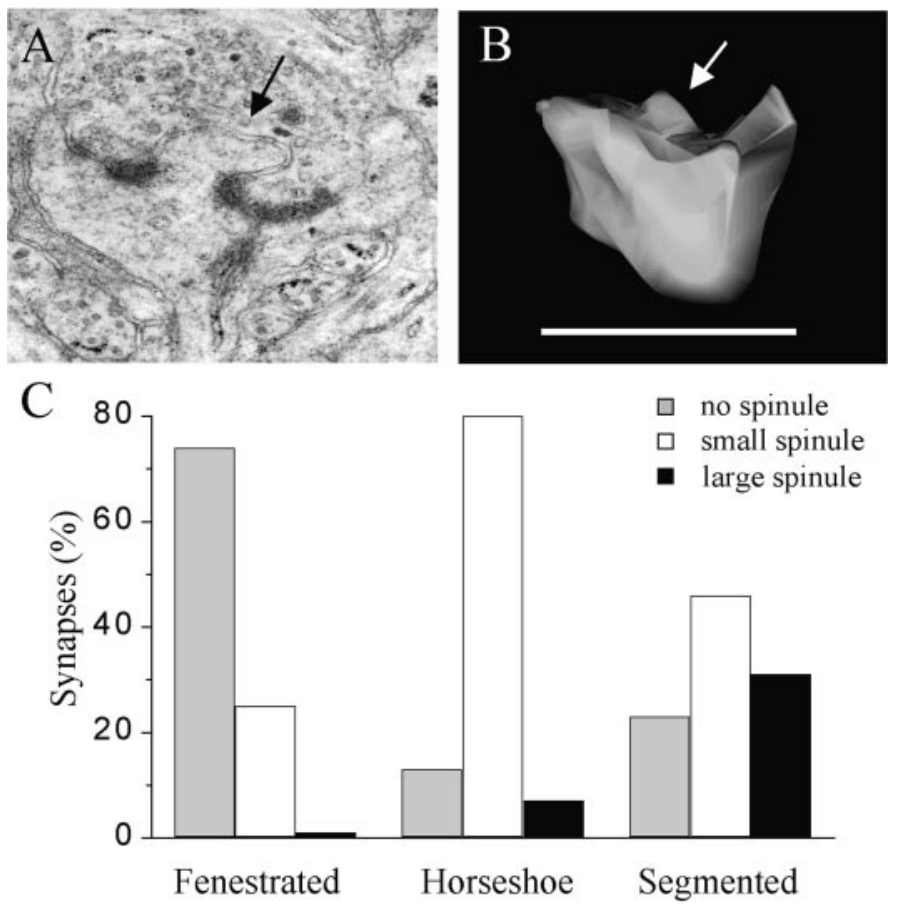

Figure 5. Presence of large spinules associated with the PSD of synapses with segmented PSDs. $A$, Illustration of a spine profile with a large spinule emerging between the two parts of the PSD. $B$, Illustration of a spinule on another reconstructed spine; arrows in $A$ and $B$ point to large spinules. Scale bar, $1 \mu \mathrm{m}$. $C$, Proportion of synapses with fenestrated, horseshoe-type, and segmented PSDs that exhibited no spinules ( gray column) or spinules of small $(<0.2 \mu \mathrm{m}$; white column) or large $(>0.2 \mu \mathrm{m}$; black column $)$ size in a population of 15,15 , and 13 reconstructed synapses, respectively.

\section{DISCUSSION}

The aim of the present study was to analyze the morphological characteristics of the three types of synapses the proportion of which has been found to change after LTP induction to investigate their possible functional role with regard to LTP mechanisms. These analyses point to three main observations. First, the transient increase in synapses with perforated PSDs found to occur $30 \mathrm{~min}$ after LTP induction essentially results from an increased proportion of synapses with segmented, fully partitioned PSDs. Second, these synapses are particular in that a much higher proportion of them contain coated vesicles and exhibit large spinules associated with the PSD, two features that strongly suggest a process of synaptic membrane remodeling. Third, the MSBs formed by duplicated spines and found 1-2 $\mathrm{hr}$ after LTP induction consist of two spines, one that is indistinguishable from simple synapses and one that is smaller and has a smaller PSD and is thus likely to be immature and newly formed. Taken together, these data support the hypothesis that LTP is associated with an increased recycling of synaptic membranes and proteins and that this process may underlie the formation of synapses with perforated PSDs and eventually lead to the formation of a new, immature spine.

The characteristics of perforated synapses have been examined in several previous studies using either single section, stereological analyses or three-dimensional reconstruction (Calverley and Jones, 1987, 1990; Geinisman et al., 1987, 1989, 1991, 1993; Harris and Stevens, 1989; Jones and Calverley, 1991; Harris et al., 1992; Itarat and Jones, 1992; Harris and Kater, 1994). Despite 

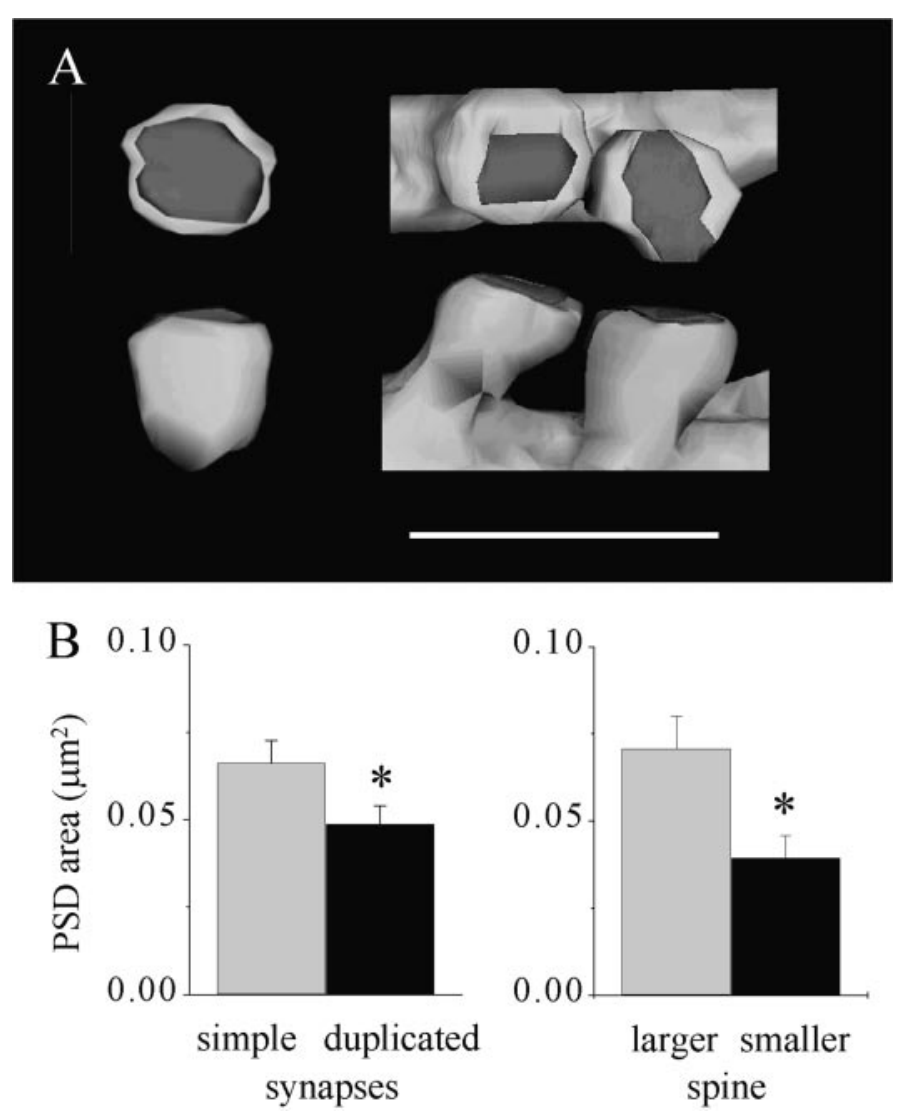

Figure 6. MSBs with two spines arising from the same dendrite include one immature spine with a small PSD. $A$, Illustration of a reconstructed simple synapse (left) and an MSB with duplicated spines (right). Scale bar, $1 \mu \mathrm{m}$. B, Left, Size of the PSD area measured in a population of 31 reconstructed simple synapses (gray column) and 30 MSBs with duplicated spines (black column). Data are the mean \pm SEM $\left({ }^{*} p<0.05\right)$. Right, Comparison of the PSD area of the larger (gray column) and smaller (black column) spines of the reconstructed MSBs with duplicated spines. Note that the larger spine has a PSD area comparable with that of a simple synapse (B, left; gray column), whereas the PSD area of the smaller spine is almost one-half that of a simple synapse $\left({ }^{*} p<0.01\right)$.

some variability, these studies showed that synapses with perforated PSDs exhibit spine heads and PSDs considerably larger than those of simple synapses, a result confirmed here. From a quantitative point of view, our data in slice cultures perfectly coincide with those reported previously by Harris et al. (1992) in acutely prepared hippocampal slices. They indicate that synapses with perforated PSDs have a spine head area and PSD area approximately three times greater than those of simple synapses. From the morphology of the PSD, three different types of synapses with perforated PSDs have been described and referred to as synapses with fenestrated, horseshoe-shaped, or segmented PSDs (Geinisman et al., 1989, 1991; Harris and Stevens, 1989; Harris et al., 1992). The size of the spine head or PSD area is not different between these three types of synapses; only the organization of the PSD changes is different. Interestingly, we found that the increase in the proportion of synapses with perforated PSDs observed 30 min after LTP induction essentially results from an increase in synapses with segmented, fully partitioned PSDs. This result is thus very consistent with the observations made previously by Geinisman et al. (1991, 1993) who also proposed that this type of synapse could be implicated in LTP. These synapses are particularly interesting for several reasons. They have large PSDs and thus probably also more receptors (Desmond and Weinberg, 1998). They exhibit two distinct PSDs that could face release sites with their own independent release probability. As reported previously (Harris and Stevens, 1989), we also observed that distinct pools of synaptic vesicles accumulate in front of each PSD. Thus an increased proportion of synapses with segmented PSDs could be equivalent to increasing the number of release sites, a mechanism that has been proposed to contribute to LTP (Geinisman et al., 1993; Edwards, 1995).

A second interesting characteristic of the synapses with segmented PSDs observed 30 min after LTP induction is that they very often contained coated vesicles; the proportion of synapses with coated vesicles was three to five times higher than that in any other type of synapse. This result was obtained using threedimensional reconstruction of a small number of spines with perforated PSDs, but it is also consistent with the single-section analyses of a large number of spine profiles (Fig. 1C). Also the distribution of coated vesicles within spines suggests that they are involved in a transfer of membrane and proteins between the spine apparatus and the synaptic membrane (Spacek and Harris, 1997). Coated vesicles were often seen fusing with the synaptic membrane, even at the level of the PSD. The frequent observation of coated vesicles in synapses with segmented PSDs is thus likely to be indicative of a process of synaptic membrane recycling and could even represent a morphological correlate of the mechanisms that lead to the incorporation of new receptors to the synaptic membrane. Strong recent evidence indeed supports the idea that expression of new receptors at the synapse plays an important role in LTP mechanisms (Lüscher et al., 2000). Coated vesicles could thus be part of the cargo system for adding or retrieving receptors to or from the synaptic membrane. Immunolabeling for glutamate receptors has indeed been associated with coated vesicles (Nusser et al., 1998). In addition to this, the recycling process evidenced by the presence of coated vesicles could contribute to the membrane expansion and increase in PSD size that probably underlie the formation of synapses with perforated PSDs.

Another observation in agreement with this interpretation is the presence of large spinules associated with the segmented PSDs of the synapses observed $30 \mathrm{~min}$ after LTP induction. The role of these spinules is also unclear, and their presence is not necessarily associated with synapses with perforated PSDs (Sorra et al., 1998). However, they may resemble in some ways the thin protrusions called filopodia that are now believed to represent precursors of spine formation (Fiala et al., 1998). Thus, spinules could also somehow reflect the existence of a process of growth and remodeling of synaptic membranes or an increased motility of spines (Matus, 2000). Together, the fact that synapses with segmented PSDs occur transiently, that they have a large spine head and large PSD, and that they contain more coated vesicles and large spinules than do the other types of synapses suggests that they represent an unstable synaptic structure undergoing a morphological remodeling. This, of course, raises the possibility that synapses with segmented PSDs could be further transformed, such as, for example, into MSBs with duplicated spines. The characteristics of MSBs with duplicated spines and particularly the fact that they include one spine with a PSD significantly smaller than that of a simple synapse suggest that the smaller spine could be immature and newly formed. Also, the striking temporal coincidence between the decrease in the proportion of synapses with segmented PSDs and the increase in the proportion 
of MSBs with duplicated spines suggests that the two types of synapses could evolve from one to the other (Toni et al., 1999).

It is tempting therefore to propose that segmented perforated synapses represent a stage of membrane expansion produced as a result of LTP induction and that this process is reversible and could evolve back either to a simple spine or, in some cases, to an MSB with duplicated spines. From a functional point of view, these morphological changes could be directly related to the increase in synaptic strength, because the process of membrane expansion that characterizes synapses with segmented PSDs includes an enlargement of the PSD area and thus probably also insertion of new receptors in the synaptic membrane (Lüscher et al., 2000). Furthermore, the formation of segmented, fully partitioned PSDs may result in the creation of two independent release sites, with their own release probabilities (Geinisman et al., 1993; Edwards, 1995). Finally this phenomenon could then be stabilized by a transformation into MSBs with duplicated spines.

In summary, the quantitative data presented here are consistent with the hypothesis that the changes in synaptic types observed after LTP reflect a process of synaptic membrane remodeling and that coated vesicles contribute to this process. Continuous monitoring of spine shape changes during LTP will be important eventually to test more directly this hypothesis.

\section{REFERENCES}

Buchs PA, Muller D (1996) Induction of long-term potentiation is associated with major ultrastructural changes of activated synapses. Proc Natl Acad Sci USA 93:8040-8045.

Buchs PA, Stoppini L, Parducz A, Siklos L, Muller D (1994) A new cytochemical method for the ultrastructural localization of calcium in the central nervous system. J Neurosci Methods 54:83-93.

Calverley RK, Jones DG (1987) A serial-section study of perforated synapses in rat neocortex. Cell Tissue Res 247:565-572.

Calverley RK, Jones DG (1990). Contributions of dendritic spines and perforated synapses to synaptic plasticity. Brain Res Brain Res Rev 15:215-249.

Carlin RK, Siekevitz P (1983) Plasticity in the central nervous system: do synapses divide? Proc Natl Acad Sci USA 80:3517-3521.

Desmond NL, Weinberg RJ (1998) Enhanced expression of AMPA receptor protein at perforated axospinous synapses. NeuroReport 9:857-860.

Edwards FA (1995) Anatomy and electrophysiology of fast central synapses lead to a structural model for long-term potentiation. Physiol Rev 75:759-787.

Engert F, Bonhoeffer T (1999) Dendritic spine changes associated with hippocampal long-term synaptic plasticity. Nature 399:66-70.

Fiala JC, Feinberg M, Popov V, Harris KM (1998) Synaptogenesis via dendritic filopodia in developing hippocampal area CA1. J Neurosci 18:8900-8911.

Fischer M, Kaech S, Knutti D, Matus A (1998) Rapid actin-based plasticity in dendritic spines. Neuron 20:847-854.

Fischer M, Kaech S, Wagner U, Brinkhaus H, Matus A (2000) Glutamate receptors regulate actin-based plasticity in dendritic spines. Nat Neurosci 3:887-894

Geinisman Y, Morrell F, de Toledo-Morrell L (1987) Axospinous synapses with segmented postsynaptic densities: a morphologically distinct synaptic subtype contributing to the number of profiles of "perforated" synapses visualized in random sections. Brain Res 423:179-188.

Geinisman Y, Morrell F, de Toledo-Morrell L (1989) Perforated synapses on double-headed dendritic spines: a possible structural substrate of synaptic plasticity. Brain Res 480:326-329.

Geinisman Y, deToledo Morrell L, Morrell F (1991) Induction of longterm potentiation is associated with an increase in the number of axospinous synapses with segmented postsynaptic densities. Brain Res 566:77-88.

Geinisman Y, DeToledo-Morrell L, Morrell F, Heller RE, Rossi M, Parshall RF (1993) Structural synaptic correlate of long-term potentiation: formation of axospinous synapses with multiple, completely partitioned transmission zones. Hippocampus 3:435-446.
Harris KM, Kater SB (1994) Dendritic spines: cellular specializations imparting both stability and flexibility to synaptic function. Annu Rev Neurosci 17:341-371.

Harris KM, Stevens JK (1989) Dendritic spines of CA1 pyramidal cells in the rat hippocampus: serial electron microscopy with reference to their biophysical characteristics. J Neurosci 9:2982-2997.

Harris KM, Jensen FE, Tsao B (1992) Three-dimensional structure of dendritic spines and synapses in rat hippocampus (CA1) at postnatal day 15 and adult ages: implications for the maturation of synaptic physiology and long-term potentiation. J Neurosci 12:2685-2705.

Itarat W, Jones DG (1992) Perforated synapses are present during synaptogenesis in rat neocortex. Synapse 11:279-286.

Jones DG, Calverley RK (1991) Perforated and non-perforated synapses in rat neocortex: three-dimensional reconstructions. Brain Res 556:247-258.

Kirov SA, Harris KM (1999) Dendrites are more spiny on mature hippocampal neurons when synapses are inactivated. Nat Neurosci $2: 878-883$

Kirov SA, Sorra KE, Harris KM (1999) Slices have more synapses than perfusion-fixed hippocampus from both young and mature rats. J Neurosci 19:2876-2886.

Korkotian E, Segal M (1999) Release of calcium from stores alters the morphology of dendritic spines in cultured hippocampal neurons. Proc Natl Acad Sci USA 96:12068-12072.

Lledo PM, Zhang XY, Sudhof TC, Malenka RC, Nicoll RA (1998) Postsynaptic membrane fusion and long-term potentiation. Science 279:399-403.

Lüscher C, Xia H, Beattie EC, Carroll RC, von Zastrow M, Malenka RC, Nicoll RA (1999) Role of AMPA receptor cycling in synaptic transmission and plasticity. Neuron 24:649-658.

Lüscher C, Nicoll RA, Malenka RC, Muller D (2000) Synaptic plasticity and dynamic modulation of the postsynaptic membrane. Nat Neurosci 3:545-550.

Maletic-Savatic M, Malinow R (1998) Calcium-evoked dendritic exocytosis in cultured hippocampal neurons. Part I: trans-Golgi networkderived organelles undergo regulated exocytosis. J Neurosci 18:6803-6813.

Maletic-Savatic M, Malinow R, Svoboda K (1999) Rapid dendritic morphogenesis in CA1 hippocampal dendrites induced by synaptic activity. Science 283:1923-1927.

Matus A (2000) Actin-based plasticity in dendritic spines. Science 290:754-758.

McKinney RA, Capogna M, Dürr R, Gähwiler BH, Thompson SM (1999) Miniature synaptic events maintain dendritic spines via AMPA receptor activation. Nat Neurosci 2:44-49.

Nieto-Sampedro M, Bussineau CM, Cotman CW (1982) Turnover of brain postsynaptic densities after selective deafferentation: detection by means of an antibody to antigen PSD-95. Brain Res 251:211-220.

Nishimune A, Isaac JT, Molnar E, Noel J, Nash SR, Tagaya M, Collingridge GL, Nakanishi S, Henley JM (1998) NSF binding to GluR2 regulates synaptic transmission. Neuron 21:87-97.

Nusser Z, Lujan R, Laube G, Roberts JD, Molnar E, Somogyi P (1998) Cell type and pathway dependence of synaptic AMPA receptor number and variability in the hippocampus. Neuron 21:545-559.

Segal I, Korkotian I, Murphy DD (2000) Dendritic spine formation and pruning: common cellular mechanisms? Trends Neurosci 23:53-57.

Shi SH, Hayashi Y, Petralia RS, Zaman SH, Wenthold RJ, Svoboda K, Malinow R (1999) Rapid spine delivery and redistribution of AMPA receptors after synaptic NMDA receptor activation. Science 284:1811-1816.

Sorra KE, Harris KM (1993) Occurrence and three-dimensional structure of multiple synapses between individual radiatum axons and their target pyramidal cells in hippocampal area CA1. J Neurosci 13:3736-3748.

Sorra KE, Fiala JC, Harris KM (1998) Critical assessment of the involvement of perforations, spinules, and spine branching in hippocampal synapse formation. J Comp Neurol 398:225-240.

Spacek J, Harris KM (1997) Three-dimensional organization of smooth endoplasmic reticulum in hippocampal CA1 dendrites and dendritic spines of the immature and mature rat. J Neurosci 17:190-203.

Stoppini L, Buchs P-A, Muller D (1991) A simple method for organotypic cultures of nervous tissue. J Neurosci Methods 37:173-182.

Toni N, Buchs PA, Nikonenko I, Bron CR, Muller D (1999) LTP promotes formation of multiple spine synapses between a single axon terminal and a dendrite. Nature 402:421-425.

Zhu JJ, Esteban JA, Hayashi Y, Malinow R (2000) Postnatal synaptic potentiation: delivery of GluR4-containing AMPA receptors by spontaneous activity. Nat Neurosci 3:1098-1106. 